\title{
Overcoming Barriers to the Remediation of Carbon Tetrachloride Through Manipulation of Competing Reaction Mechanisms
}

\author{
Paul G. Tratnyek \\ Department of Environmental \\ and Biomolecular Systems \\ OGI School of Science \& Engineering \\ 20000 NW Walker Road \\ Beaverton, OR 97006-8921
}

\section{Batch Experimentation Objective:}

Quantify the kinetics of all competing product-formation pathways, over a range of conditions relevant to groundwater remediation, using well-mixed batch reactors and analysis primarily by chromatography. At OGI, batch experiments were conducted on $\mathrm{Fe}(0)$ systems (both Fisher Electrolytic and Nano-sized iron). The experiments were done with and without buffer. The buffered experiments tried to contrast two buffers: an organic buffer (EPPS, presumably a $\mathrm{H}$ atom donor), and the inorganic borate. In the buffered experiments, the $\mathrm{pH}$ was varied (7.3 and 8.4). For the pre-exposure treatment, after trying a variety of methods, like shaking and not shaking for varied amounts of time, it was decided to stick with not shaking and have a pre-exposure of 24 hours. The unbuffered data did not show any marked trend with increasing mass of Felc. However, $3.5 \mathrm{~g}$ of Fe showed about $100 \%$ conversion to $\mathrm{CHCl}$, and $1 \mathrm{~g}$ of Fe showed $50 \%$ conversion. At pHs 8.4 and 7.3, there was no trend observed for branching ratios between EPPS and Borate buffer. kCT (disappearance rate constant of carbon tetrachloride) values were found to be different from $\mathrm{CT}$ and CF fits. Experiments with nano-iron (unbuffered, buffered with both buffers at $\mathrm{pH}$ 8.3), did not show any trend with respect to Fisher Iron, except for the unbuffered experiments, where the CF ?yield? was less in the nano iron case. Future experiments involve testing for chloride, formate and CO, and performing experiments over a wider range of $\mathrm{pH}$ and buffers. Batch experiments were conducted at PNNL to compare the efficiency and product distribution of representative $\mathrm{Fe}(\mathrm{II})$ and $\mathrm{Fe}(0)$ systems applied to dechlorination of CCl4. These experiments involved 1) a smectite clay with Fe(III) in its structure that had been reduced to $\mathrm{Fe}(\mathrm{II})$ by dithionite treatment, 2) the same clay to which $\mathrm{Fe}(\mathrm{II})$ was added as an exchangeable cation, 3) electrolytic $\mathrm{Fe}(0)$ from Fisher, and 4) a mixture of the reduced clay and $\mathrm{Fe}(0)$. Experiments were conducted in headspace vials at $\mathrm{pH} 7$ in either bicarbonate or bis-tris propane buffers. Reactant and product concentrations were determined by headspace analysis using GC/MS. Results from the first run of this experiment showed relatively little dechlorination by the Fe(II) system, and from 50$80 \%$ dechlorination by the $\mathrm{Fe}(0)$ system after $48 \mathrm{~h}$. Essentially no $\mathrm{CHCl} 3$ was seen in the Fe(II) system, whereas as $30 \%$ of the original CCl4 was converted to $\mathrm{CHCl} 3$ in the $\mathrm{Fe}(0)$ system. Very low amounts of $\mathrm{CH} 2 \mathrm{Cl} 2$ were seen in all treatments. Plans are underway to repeat this experiment and use a cryo-GC capability that will allow simultaneous determination of $\mathrm{CO}$ as well as the chlorinated methanes. This is important because $\mathrm{CO}$ represents the end product of the second hypothetical dechorination pathway that may compete with the $\mathrm{CHCl} 3$ pathway and will aid in mass balance calculations. An additional experiment to evaluate the Henry's Law constant for CCl4 in aqueous solutions in the presence and absence of clay showed no significant difference due to the presence of clay, although slightly higher gas-phase concentrations were seen when clay was present.

\section{Theoretical Modeling:}

Calculate the electronic structure and properties of all relevant contaminant species and intermediates using ab initio quantum mechanical models, and use these results to characterize the controls on branching among reaction products. Ab initio molecular modeling at PNNL has calculated thermochemical properties for all species relevant to the putative mechanisms of environmental degradation of carbon tetrachloride. Next we will investigate activation barriers and synthesize these results to evaluate the feasibility of each step of each reaction mechanism. 